\title{
Managing Split-attention and Redundancy in Multimedia Instruction
}

\author{
SLAVA KALYUGA, PAUL CHANDLER* and JOHN SWELLER \\ University of New South Wales, Australia
}

\begin{abstract}
SUMMARY
Two experiments investigated alternatives to split-attention instructional designs. It was assumed that because a learner has a limited working memory capacity, any increase in cognitive resources required to process split-attention materials decreases resources available for learning. Using computer-based instructional material consisting of diagrams and text, Experiment 1 attempted to ameliorate split-attention effects by increasing effective working memory size by presenting the text in auditory form. Auditory presentation of text proved superior to visual-only presentation but not when the text was presented in both auditory and visual forms. In that case, the visual form was redundant and imposed a cognitive load that interfered with learning. Experiment 2 ameliorated split-attention effects by using colour coding to reduce cognitive load inducing search for diagrammatic referents in the text. Mental load rating scales provided evidence in both experiments that alternatives to split-attention instructional designs were effective due to reductions in cognitive load. Copyright (C) 1999 John Wiley \& Sons, Ltd.
\end{abstract}

The limitations of working memory have been well know since Miller (1956). Only a few elements of information can be processed in working memory at any time. Too many elements may overburden working memory, decreasing the effectiveness of processing. In contrast to working memory, an unlimited number of elements can be held in long-term memory in the form of hierarchically organized schemas which permit us to treat multiple sub-elements of information as a single element categorized according to the manner in which it will be used. Schemas allow us not only to store learned information in long-term memory but, because multiple elements of information are treated as a single element in working memory, schemas also reduce the burden on working memory.

As a consequence of this architecture, any increase in information processing not directly related to the acquisition of new schemas inevitably consumes part of available working memory capacity, decreasing resources available for learning. Cognitive load theory, incorporating this architecture, has been used to develop a number of instructional procedures designed to optimize limited working memory (e.g. see Sweller, 1994; Sweller, van Merrienboer and Paas, 1995). The

*Correspondence to: Paul Chandler, School of Education Studies, University of New South Wales, Sydney, NSW, 2052, Australia. E-mail: p.chandler@unsw.edn.au

Contract grant sponsor: Australian Research Council.

CCC 0888-4080/99/040351-21 \$17.50

Copyright (C) 1999 John Wiley \& Sons, Ltd.

Accepted 16 April 1998 
theory assumes that information presented to learners and the activities required of them should be structured to eliminate any avoidable load on working memory and to maximize the acquisition of new schemas.

For example, many conventional instructional materials require learners to unnecessarily split their attention between diagrams and text. To understand a conventional separate text and diagram format, the learner must hold small segments of text in working memory while searching for the matching diagrammatic entity, with this ongoing process continuing until all the information is rendered intelligible. It should be noted that this process of mental integration is not directly related to learning; it is essentially a preliminary activity to learning. An alternative instructional format is to have related information physically located together in order to avoid extensive search and match behaviour. Research has indicated that instructions involving diagrams and text that need to be mentally integrated to be understood should be restructured into physically integrated formats with as small a number of units as possible (see Chandler and Sweller, 1991, 1996; Kalyuga, Chandler and Sweller, in press; Mayer, 1989; Mayer and Gallini, 1990; Tarmizi and Sweller, 1988). Physical integration of related elements of diagrams and text reduces working memory load and enhances learning. This split-attention effect was obtained in most previous studies using direct spatial integration of separate text and diagrams. Fragments of text were directly embedded into the diagram in close proximity to corresponding components of the diagram. Arrows directed from the text to the corresponding elements of the diagram were used to make the search process easier for learners.

It should be noted that integration of diagrams and text is required if these sources of information are unintelligible in isolation for a learner. If individual sources of information are self-contained with, for example, the text merely redescribing information contained in a self-contained diagram, integration of the redundant information with essential information imposes a cognitive load that interferes with the learning process. In this situation the elimination, rather than integration, of redundant sources of information is beneficial for learning. This redundancy effect has been demonstrated in a number of previous studies using diagram-and-text instructional presentations (Bobis, Sweller and Cooper, 1993; Chandler and Sweller, 1991; Kalyuga, Chandler and Sweller, 1998; Sweller and Chandler, 1994).

Two experiments reported in this paper were designed to investigate some alternative ways of circumventing the problems associated with split-attention. One of these techniques is based on the assumption that working memory consists of multiple, partly independent processors, with, for example, separate processors for auditory and visual information (Baddeley, 1992; Penney, 1989; Schneider and Detweiler, 1987). Baddeley (1992) proposed a model including three stores: a phonological loop, a visuo-spatial sketch pad, and a central executive. The phonological loop processes auditory information (verbal material in an auditory form), while the visuo-spatial sketch pad deals with visual information such as diagrams and pictures. Pavio's (1990) dual coding theory also suggests that information can be encoded, stored and retrieved from two distinct systems, one suited to verbal information, the other to images. The two systems are interconnected and may both contribute to memory performance. If information is coded in both the verbal and imaginal coding systems, memory for the information may be enhanced. Penney (1989) proposed a model of working memory (the 'separate stream hypothesis') where the processing of auditory and visually 
presented verbal items was carried out independently by auditory and visual processors in working memory. She provided a considerable body of research in support of this hypothesis.

According to cognitive load theory, learning might be inhibited when learners must split their attention between and mentally integrate text and graphics because the integration process might overburden limited working memory capacity. However, when textual information is presented in auditory form, mental integration with a diagram may not overload working memory because working memory may be enhanced by the use of both visual and auditory channels. Such a dual mode of presentation might be used to circumvent cognitive load problems caused by splitattention. This phenomenon will be referred to as the instructional modality effect.

It should be pointed out that dual-mode presentations do not reduce extraneous cognitive load, but rather increase effective working memory capacity. The amount of information that can be processed using both auditory and visual channels might exceed the processing capacity of a single channel. Thus, limited working memory may be effectively expanded by using more than one sensory modality, and instructional formats in which separate sources of information (otherwise requiring integration) are presented in alternate, auditory or visual, forms might be more efficient than equivalent single-modality formats.

In a series of experiments using geometry instructional material, Mousavi, Low and Sweller (1995) found that a visually presented geometry diagram, combined with aurally presented statements, enhanced learning compared to a conventional, visualonly presentation. In a split-attention situation, increasing effective working memory by using more than one modality produced a positive effect on learning, similar to the effect of physically integrating separate sources of information. Tindall-Ford, Chandler and Sweller (1997) also investigated this effect using elementary electrical engineering instructions and showed that an audio text/visual diagram format was superior to purely visually based instructions. Measures of subjective mental load and instructional effectiveness estimates (see Paas and Van Merrienboer, 1993, 1994) were used to support the suggestion that the effect is due to cognitive load factors.

Jeung, Chandler and Sweller (1997) demonstrated that the additional processing capacity provided by using dual-mode (audio/visual) presentations enhanced learning only if mental resources were not devoted to extensive visual search involved in the coordination of auditory and visual information. Experimenting with primary school, computer-based geometry instructional materials, they found that if visual search was high, then audio-visual instruction was only beneficial if visual indicators in the form of electronic flashing were incorporated into the instructional format. In this case, a standard audio/visual format without the aid of flashing proved no better than visualonly instruction. In contrast, when instructional materials low in visual search were used, a standard audio/visual format resulted in superior learning to a visual-only format. There was no beneficial effect of electronic flashing, suggesting that the effectiveness of visual indicators depended on the cognitive load imposed by visual search.

Mayer and his associates (see Mayer, 1997) have conducted a number of experiments demonstrating the superiority of audio/visual instructions. Mayer and Anderson (1991) presented information on how a bicycle tyre pump works. There were four experimental conditions in this study. The first group viewed an animation depicting the operation of a bicycle tyre pump and listened to simultaneous audio text; the second group was given only the audio text without the animation; the 
third group was provided only the animation with no audio component; and the fourth group received no formal training (control group). Results of this experiment (measured by number of creative and detailed solutions on the problem-solving test) demonstrated that the first group outperformed the other three groups. This research was not concerned with split-attention factors. Further research (Mayer and Anderson, 1992; Mayer and Sims, 1994) demonstrated that audio/visual instructions may only be superior when the audio and visual information are presented simultaneously rather than sequentially. (Mayer and his colleagues called this phenomenon the 'contiguity effect'.) This effect could be interpreted within a cognitive load approach as providing an example of the split-attention effect.

The aim of the first experiment of this paper was to confirm that a dual-mode presentation of instructional information can be used as an alternative to physical integration in circumventing the problems associated with split-attention. Computerbased multimedia instructions on soldering theory were used in this experiment. Textual explanations were presented to one of the experimental groups in auditory mode simultaneously with animations of corresponding parts of visually presented diagrams. Instructional materials presented to another experimental group used auditory explanations simultaneously with the same visually presented text and animated diagrams. This instructional format is widespread in many lecturing situations. Frequently, identical visual and auditory text is used when referring to a diagram or other illustration. However, such duplication of textual information using different modes of presentation was expected to be redundant and increase the risk of cognitive overload. A third group, presented with written text and diagrams, acted as the control.

As mentioned above, the split-attention effect was obtained in previous studies using direct embedding of textual explanations into a diagram in close proximity to corresponding components of the diagram in order to reduce unnecessary search between diagrams and text. It is reasonable to suggest that colour-coding of the corresponding elements of the text and diagram should produce a similar effect of reducing search and its associated cognitive load. Colouring elements of a diagram in the same colours as corresponding textual elements should reduce an unnecessary working memory load by reducing search processes involved with split source instructional formats. The released cognitive resources could be devoted to schema acquisition. The aim of the second experiment was to demonstrate that colour-coding also could be used as a technique to circumvent the cognitive load problems associated with split-attention. Computer-based instructions in elementary electrical engineering were used in this experiment. Elements of a wiring diagram were coloured in the same unique colours as corresponding elements of textual explanation. It was suggested that this technique should reduce an unnecessary working memory load by reducing search processes involved with split-source instructional formats.

\section{EXPERIMENT 1}

Experiment 1 was designed to investigate if the instructional modality effect could be used to circumvent the cognitive load associated with split-attention. Consider the diagram and text of Figure 1. The figure depicts a fragment of computer screen for instruction on the Fusion Diagram used to represent states (solid, plastic, or liquid) of 




Figure 1. Visual text instructional format for the Fusion diagram

tin/lead solder depending on its content (tin/lead ratio) and temperature. It is used to determine the most appropriate solder for specific needs.

For example, to reduce disturbance of the joint which may result in a low-strength connection, solder should pass through the plastic state as quickly as possible when cooling from the liquid state. For this purpose, 63/37 solder (containing 63\% tin and $37 \%$ lead) would be the most appropriate. This solder is called eutectic solder. It passes between the liquid and solid states directly without passing through the plastic state. When heated, this solder becomes liquid at lower temperature than all other solder ratios. It is important for reducing the risk of heat damage to the components during soldering. On the other hand, if a specific technological process requires solder to stay in the soft plastic state as long as possible when heating from the solid state or cooling from the liquid state, the solder containing about $20 \%$ tin would be more appropriate.

Assume the material of Figure 1 (explaining a feature of $60 / 40$ solder) is presented to learners who have just assimilated the basic concepts required to understand the information. For example, they may know that the vertical axis represents the range of temperatures, and the horizontal axis represents the tin/lead ratios. They may also know that the curves on the diagram represent the borders between correspondent states of solder. Nevertheless, they do not know how to use this diagram to determine characteristics of specific solders (in other words, they have not acquired schemas for using the Fusion Diagram). For these learners to understand the information, both the diagram and the text must be mentally integrated. Because many interacting visual elements of information are involved in such integration (e.g. 60/40 ratio, liquid state, plastic state, cooling that corresponds to a downward move along 
the ratio line, the borders of the plastic state), a heavy cognitive load could be expected. This combination of learners and instructional materials represents an example of split-attention.

Cognitive load theory suggests that one way to facilitate learning may be to physically integrate the two sources of information to reduce the need for mental integration (e.g. by directly embedding portions of text into the diagram). An alternative to physical integration may be to increase effective working memory capacity by using a dual-mode presentation technique and replacing the visual textual explanations with equivalent auditory explanations. The diagram and the auditory explanation still must be mentally integrated, with many interacting audio and visual elements of information involved in the integration but, due to the effective expansion of working memory because of the combined use of visual and auditory processors, the material may be more readily handled than when presented solely in a visual only format.

Finally, assume that the instructional presentation in Figure 1, including the text, is used simultaneously with an oral narration of the text. In this situation, either the auditory commentary or the written text is redundant. All the interacting elements of the diagram and visual and auditory explanations must be mentally integrated. Working memory capacity, even if expanded due to the use of both audio and visual processors, should be more readily able to handle the material without the written text. Establishing connections between corresponding elements of visual and auditory components of working memory may unnecessarily consume additional cognitive resources to those used in a diagram plus auditory text alone format.

Thus, from the point of view of cognitive load theory, a duplication of information using different modes of presentation increases the risk of overloading working memory capacity and may have a negative effect on learning. One of the duplicated sources of information (visual or auditory explanations) is clearly redundant. It does not provide any new information for the learners in addition to that already provided. In this situation, elimination of a redundant (duplicated) source of information might be beneficial for learning.

The following three instructional formats of computer-based multimedia instructions on the Fusion Diagram were compared in this experiment: Visual text, Audio text, and Visual plus Audio text. For the first two formats, we expected to replicate the modality effect of previous studies (Jeung, Chandler and Sweller, 1997; Mousavi, Low and Sweller, 1995; Tindall-Ford, Chandler and Sweller, 1997) with Audio text being superior to Visual text. For the third format, it was expected that inclusion of the visual text simultaneously with the same text in an audio form would impose an additional cognitive load due to redundancy, thus interfering with learning.

\section{Method}

\section{Participants}

Thirty-four first-year trade apprentices and trainees from a Sydney company participated in the experiment. All apprentices had very limited or no practical experience with soldering. None of the apprentices had any previous exposure to instructions on the Fusion Diagram and its implications for soldering. All participants had completed at least Year 10 of high school and were half-way through the first-year of a four-year trade course. The instructional materials presented to the participants of Experiment 1 were part of the soldering module of the first-year training programme. 


\section{Materials and procedure}

Participants were randomly allocated to one of the three groups (12 learners in the Visual plus Audio text group, 11 learners in the Visual text group, and 11 learners in the Audio text group) corresponding to the three formats of instructional materials. The experiment was entirely computer based allowing electronic presentation of all training materials as well as automatic recording of all data (learners' responses, number of reattempts at exercises, etc.) using Authorware Professional 3.0 for Windows. All participants were tested individually, and all tests were conducted in a single session. Each participant spent about one hour at a monitor (with breaks as needed).

The experiment consisted of two instruction phases and two test phases. The first instruction phase included a brief general introduction to the Fusion Diagram (e.g. explanations of what parameters are represented by the horizontal and vertical axes; borders between states represented by the curves). The materials were presented in three formats (Visual plus Audio text, Visual text, Audio text) to corresponding instructional groups. The Visual plus Audio text format contained sequentially introduced animated components of the Fusion Diagram (axes, curves, different areas of the diagram, etc.) with written explanations of newly appearing elements. The same explanations were simultaneously presented in an auditory format (via headphones) concurrently with corresponding animations. The Visual text format was visually identical to the Visual plus Audio text format with all the auditory explanations eliminated (the headphones were removed). The Audio text differed from the Visual plus Audio text format by the elimination of any written (e.g. visual) explanations of the diagram (the headphones were turned on).

All three computer-based presentations were self-paced. Seven interactive exercises were incorporated into all the computer presentations with immediate feedback and built-in 'hints'. The interactive exercises served two purposes. First, the number of reattempts at each exercise was used to differentiate between instructional groups. Second, the exercises and accompanying hints ensured that all participants had sufficient knowledge of the basic principles of the Fusion Diagram before proceeding to the second instructional phase. Levels of specificity of the 'hints' increased with the number of reattempts at responding to a specific question. The interactive exercises consisted of six multiple-choice items, and one question requiring apprentices to drag labels to their proper places on the diagram. Numbers of reattempts at exercises were recorded. The set of interactive exercises separated the two instructional phases. The number of reattempts provided the data for analysis in the first instructional phase. No other data were collected at this stage because of the purpose of the first instruction phase and interactive exercises was to provide learners with the very basic knowledge of the Fusion Diagram necessary for understanding the main instructional materials that followed.

During the second instruction phase, the learners studied descriptions of major features of the $60 / 40$ and eutectic solders presented using the same computer-based techniques as those used during the first instruction phase. The descriptions were displayed in the same three formats (Visual diagram/Visual text plus Audio text, Visual diagram/Visual text, Visual diagram/Audio text) and the participants remained in their instructional groups.

After each participant completed studying the instructions, they were asked to rate the subjective mental load associated with learning the instructional materials 
(see Paas and Van Merrienboer, 1993, 1994). Ratings were collected on a seven-point Likert-type scale with participants being asked to estimate how easy or difficult the instructions were to understand by clicking one of the seven choices on the screen from extremely easy (corresponding to the score 1) to extremely difficult (corresponding to the score 7). The results obtained from this rating scale were used as an indicator of cognitive load (the higher the score, the higher the estimated mental load).

The collection of mental load ratings was followed by a test phase. The first part of the test phase consisted of a fault-finding task. All participants were presented with a faulty 'fusion diagram' with six elements in incorrect positions. The participants were asked to identify the faults on the diagram by clicking any element in an incorrect position. They also were warned that clicking on any element which was not faulty would decrease the score. This was done in order to prevent participants from random clicking of any screen elements. Up to 60 seconds were allowed for this task with the remaining time indicated on the screen. The fault-finding exercise was transfer task designed to measure trainees' understanding of soldering instructions.

The second part of the test consisted of five multiple-choice questions relating to 60/40 and eutectic solders. The Fusion Diagram was presented on the screen. The questions were directly concerned with the features of solders which were described previously (e.g. At what temperature range is the 60/40 solder in the plastic state? Why is the eutectic solder the most appropriate for using in difficult production conditions? Which solder from those listed below becomes liquid at a lower temperature?). Up to six options were provided for each multiple-choice item. Each item had only one correct response and was judged as either correct or incorrect, providing a total score out of 5 for the second part of the test. Up to 45 seconds were allowed for each multiple-choice item with the remaining time indicated on the screen

\section{Results and discussion}

The variables under analysis were number of reattempts at exercises after the first instruction phase, subjective ratings of mental load, and test performance scores on fault finding and multiple-choice tasks. Means and standard deviations are displayed on the charts of means in Figure 2 (upper numbers in bold inside each bar represent means, lower numbers in plain type represent standard deviations).

One-way analyses of variance (ANOVA) indicated significant differences (the 5\% level of significance is used throughout this paper, unless otherwise stated) on all the variables: number of reattempts of exercises during the first test phase, $F(2,29)=3.58$, $M S e=20.52$. (The number of reattempts for two participants, both from the Visual plus Audio text group, were missed due to software problems. Thus, the significant difference for number of reattempts was based on 10 learners in the Visual plus Audio text group, 11 learners in the Visual text group, and 11 learners in the Audio text group); subjective rating scale data, $F(2,31)=4.32, M S e=1.68$; fault-finding scores, $F(2,31)=4.86, M S e=1.97$; question-answering scores, $F(2,31)=7.80, M S e=$ 1.36. Post-hoc comparisons using Newman-Keuls contrasts produced significant differences between the Audio text and Visual plus Audio text groups for all the above dependent variables, and between the Audio text and the Visual text groups for the test performance scores on fault-finding and multiple-choice tasks. In all cases, means favoured the Audio text group with the subjective rating scores indicating that this group found its instructions easier to learn. 

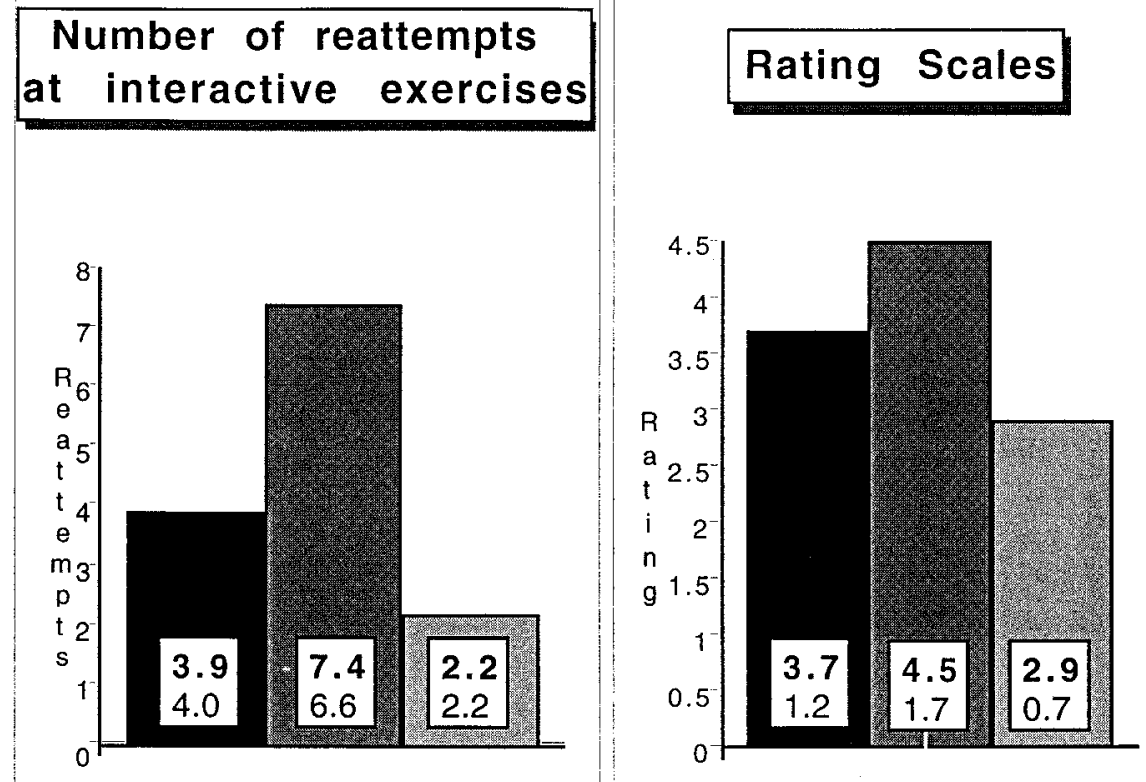

\section{Fault-finding Scores}
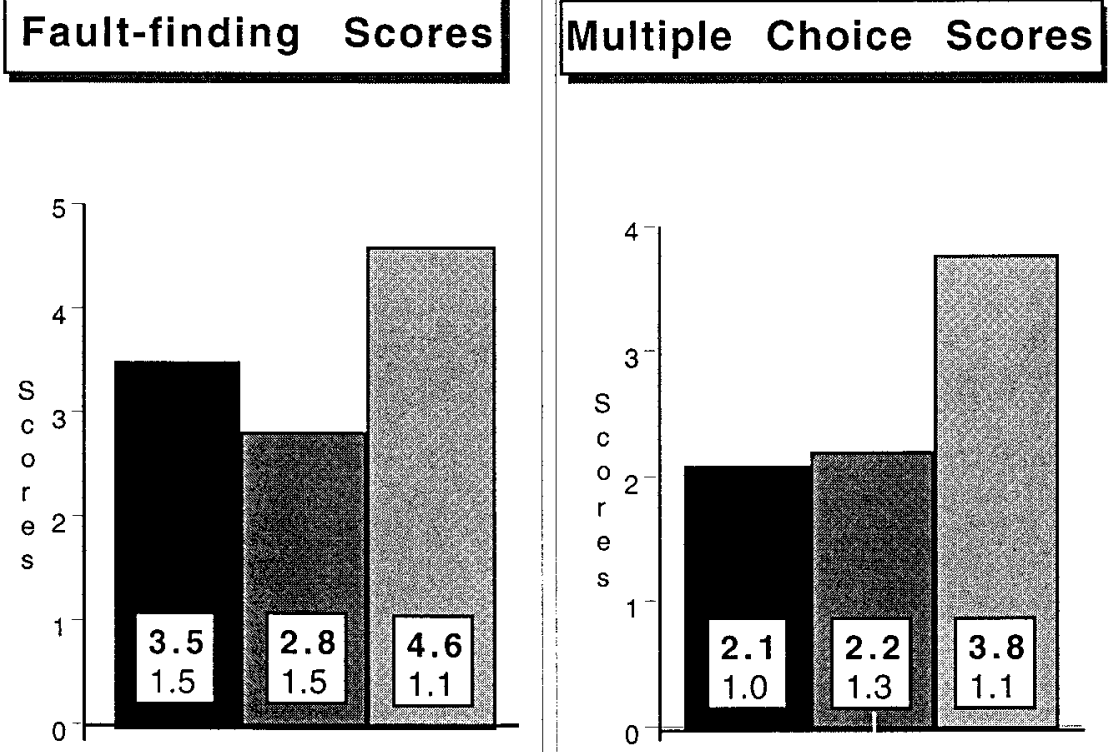

Figure 2. Charts of means for the data of Experiment 1 
Instructional effectiveness measures were calculated using Paas and Van Merrienboer's $(1993,1994)$ procedure. This approach allows measures of cognitive load (obtained by participants' subjective ratings) to be combined with measures of performance (obtained from the two measures of test performance-fault-finding and multiple-choice responses) in order to derive information on the relative effectiveness of instructional conditions and estimate the cognitive cost of instruction. High effectiveness occurs under conditions of low cognitive load and high test performance and low effectiveness under high cognitive load and low test performance. Effectiveness values can be calculated by converting cognitive load and performance measures into $Z$-scores ( $R$ and $P$, respectively) and combining those scores using the formula:

$$
E=(P-R) / / S Q R T(2) /
$$

In order to depict effectiveness, the cognitive load $Z$-scores $(R)$ and performance $Z$-scores $(P)$ are represented in a cross of axes (see Figure 3 ). In this coordinate system, the relative effectiveness of an instructional condition as a point $(R, P)$ on the diagram can be measured as the distance from this point to the line of zero effectiveness $(E=0)$ and calculated using the above formula. The high-effectiveness area (relatively lower cognitive load with higher performance) is above the line $E=0$. The low-effectiveness area (higher cognitive load with lower performance) is located below this line.



Figure 3. Representation of relative condition effectiveness 
Figure 4 demonstrates the results of the application of this procedure. Measures of cognitive load were obtained by participants' ratings of subjective mental load. Faultfinding and multiple-choice items were used as measures of performance. The results for the Audio text group are located in the high-effectiveness area (relatively lower cognitive load with higher performance), whereas the results for the other two groups are located in the areas of relatively lower effectiveness (more cognitive load with lower performance).

Analysis of variance yielded significant differences in effectiveness between the groups on the fault-finding task, $F(2,31)=7.74, M S e=0.97$, and multiple-choice task, $F(2,31)=10.66, M S e=0.79$. Newman-Keuls tests indicated significant differences in the predicted direction between the Audio text and both the Visual

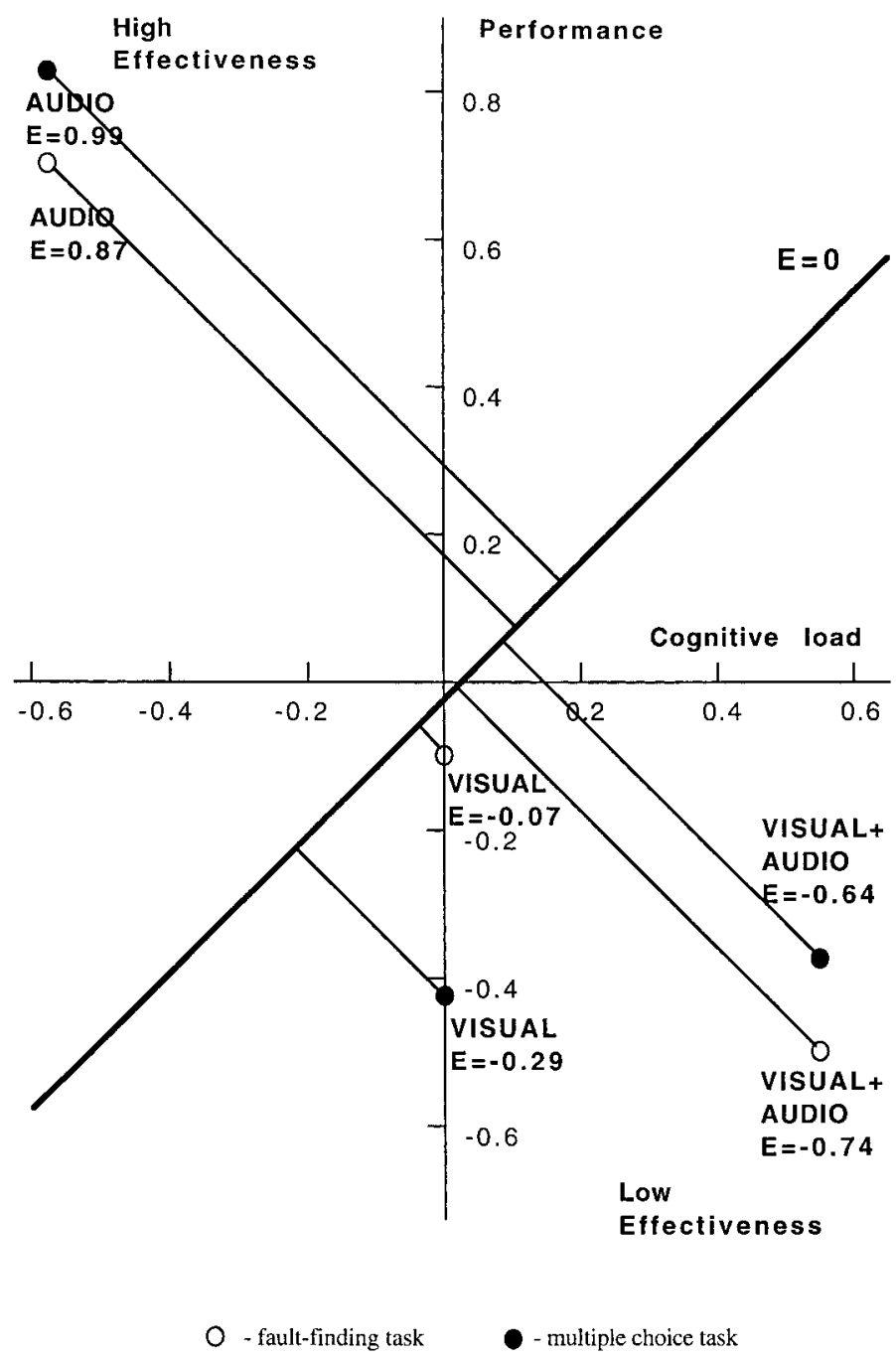

Figure 4. Representation of relative condition effectiveness $(E)$ for Visual text (VISUAL), Visual plus Audio text (VISUAL + AUDIO) and Audio text (AUDIO) groups for the Fusion Diagram test 
text and Visual plus Audio text groups for the test performance scores on fault-finding and multiple-choice items. The instructional conditions (in terms of cognitive cost) for the Audio text group were significantly more effective than for both the Visual text and Visual plus Audio text groups, as was predicted by cognitive load theory.

The results of this experiment provided evidence that the modality effect might be used to circumvent the cognitive consequences of split-attention. Dual-mode instructional presentations of text and diagrams do not eliminate split attention, but rather, increase available working memory capacity by combining, in part, the capacity of visual and auditory memory channels. As a result, learners can more efficiently handle split-attention without a cognitive overload and negative learning effects.

The redundancy effect was also obtained in this experiment (the Audio text group outperformed the Visual plus Audio text group). Having auditory textual explanations duplicated in a visual form significantly inhibited learning due to an increase in cognitive load. In fact, the dual-mode duplication format was the worst of the three instructional procedures. Redundancy overrode all the benefits of dual-mode presentation. While other explanations may be offered for the negative effects of simultaneous visual and auditory presentations, the employment of cognitive load measures provides evidence that the result was consistent with a redundancy hypothesis.

The redundancy effect obtained in this experiment can be related to the results of Schooler and Engstler-Schooler (1990) on verbal overshadowing of visual memories. They found that having to verbalize a visual stimulus (e.g. describing facial features of a person seen on a videotape segment) impaired subsequent recognition performance. It may be assumed that the requirement to verbalize was redundant, imposed an extraneous cognitive load and so interfered with learning.

It is widely believed (including among many multimedia instructional designers and educational software manufacturers) that duplicating informationally identical audio and visual material facilitates learning. The results of this experiment suggest that dual-mode presentation under such circumstances has negative rather than positive effects. Dual-mode presentation only is effective when the two modes present different information that must be mentally integrated before it can be understood.

\section{EXPERIMENT 2}

Experiment 1 used the modality effect to circumvent the problems associated with split-attention. Experiment 2 used a different technique to negate the same problems. Rather than increasing effective working memory by using dual-modality presentation techniques as was used in Experiment 1, a new procedure to reduce the cognitive load caused by split-attention was devised. Figure 5 represents an example of a conventional, split-source instructional format on the operation of a push-button, on/off, light-switching circuit (the Starter configuration). For novice learners neither the diagram nor the text are understandable in isolation. To understand the information, both the diagram and the text must be mentally integrated. For all the elements of information included in the text, corresponding related elements of the diagram must be found and interactions between them traced. Because many interacting elements of information are involved in such search and tracing (e.g. circuit breaker, start and stop push-buttons, coil, light, switch), a heavy cognitive load due to split-attention can be expected. 


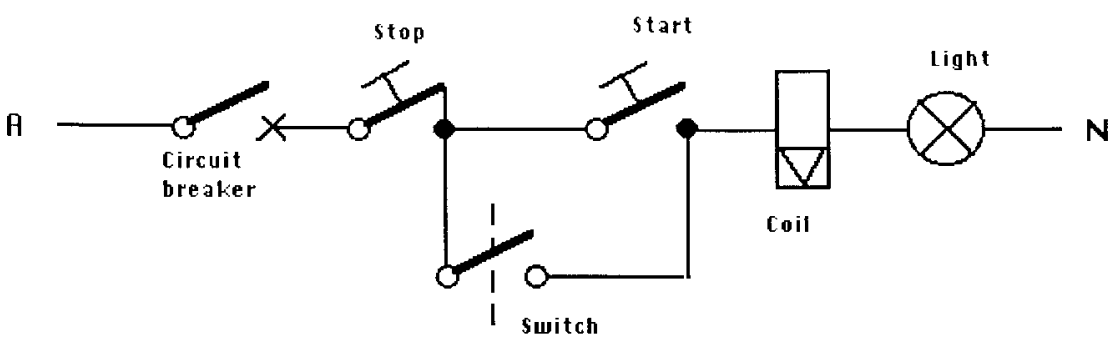

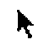

1. Current flows only through a completely closed circuit

2. The first step towards producing a closed circuit is closing the circuit breaker

3. Pressing down the start button closes the circuit and allows the current to flow through to the coil and light. The light is operational

4. The energised coil closes the switch

5. Closing switch provides an alternative closed circuit for the coil to that prouided by the start button. This circuit acts as a holding one: thestart button now can be released without breaking the current flow through the coil and light

6. To cease operation of the light the stop button is pressed. The circuit is now open, the coil is no longer energised and the switch returns to its normal open position. The stop button now can be released

Figure 5. Conventional format of instructional presentation explaining operation of the Starter

Learning may be facilitated in this situation by increasing effective working memory capacity using dual-mode presentation techniques, as in Experiment 1, or by physically integrating the two sources of information thus reducing the cognitive load associated with mental integration. Physical integration is effective because it reduces search for corresponding elements. Colouring elements of a diagram in the same unique colours as corresponding textual elements may similarly reduce cognitive load by reducing search.

Colour-coding was used to reduce search in the material of Figure 5. By clicking on any paragraph in the text, a learner could see all the electrical elements mentioned in this paragraph in the same unique colours in the diagram and text. In the example of Figure 5, if section 3 of the textual explanations has been selected, it results in critical aspects of that section and the relevant parts of the diagram assuming the same unique colours. The word 'start' in the text turns blue as does the start-button in the diagram; the word 'coil' in the text turns green as does the coil in the diagram, etc. It was expected that colour-coding might reduce an unnecessary working memory load by reducing search processes involved with split-source instructional formats. The released cognitive resources can then be devoted for meaningful learning of the circuit operation.

Experiment 2 was designed to test this hypothesis using computer-based instructions in elementary electrical engineering. The experiment was designed to compare conventional separate-diagram-and-text and colour-coded-diagramand-text instructional formats using participants without any substantial knowledge of elementary electrical engineering. It was predicted that a colour-coded 
instructional format would be superior to a conventional format thus providing evidence that colour-coding could be used to reduce the negative consequences of split-attention.

\section{Method}

\section{Participants}

Sixteen trainees from a Sydney company without any substantial knowledge of electrical circuits participated in this experiment. All participants had completed at least Year 10 of high school and had just commenced their first-year trade course. All trainees had a basic understanding of the electrical concepts which were necessary to understand the to-be-taught material. The instructional materials presented to the participants in Experiment 2 formed a part of the industrial wiring component of the first-year electrical training programme.

\section{Materials and procedure}

Participants were randomly divided into two groups: colour-coded-diagram-and-text and conventional separate-diagram-and-text groups. Eight participants were allocated to each of the two groups. The study was entirely computer based allowing electronic presentation of all training materials as well as automatic recording of all data (using Authorware Professional 3.0 for Macintosh). The participants were asked to study a simple electrical circuit for switching a light on/off by pushing start/stop buttons. The conventional format (Figure 6) included the electrical circuit with a textual explanation located below the circuit. The colour-coded-diagram-and-text format consisted of the identical diagram and text as the conventional format. The difference was that by clicking on any paragraph in the text, a learner could see all the electrical elements mentioned in that paragraph in the same unique colours in the diagram and text. The learners studied the instructions at their own pace without any time restrictions. All participants were tested individually, and all tests were conducted in a single session.

After each participant completed studying this circuit they were asked to rate the subjective mental load associated with learning the instructional materials. Ratings were collected on a seven-point scale with participants being asked to estimate how easy or difficult a circuit was to understand by clicking one of the seven choices ranging from 1 (extremely easy) to 7 (extremely difficult).

The test comprised two parts. The first part consisted of a fault-finding task. The learners were presented with a faulty diagram of the circuit, with six elements being incorrect or in the incorrect position. Each participant was asked to find the faults and click on all faulty elements. As in Experiment 1, to avoid random clicking, trainees were warned that clicking on any element which was not faulty would decrease the score. Up to 60 seconds were allowed for this task with the remaining time indicated on the screen.

The fault-finding task was followed by a multiple-choice task. This consisted of five items relating to questions about the operation and function of the circuit. Only the circuit diagram identical to the instructional phase diagram was available during this part of the test. Each multiple-choice item had up to four options and trainees were required to click the correct response. Each item was judged as either correct or incorrect. The first four questions were specific questions relating to the function of 


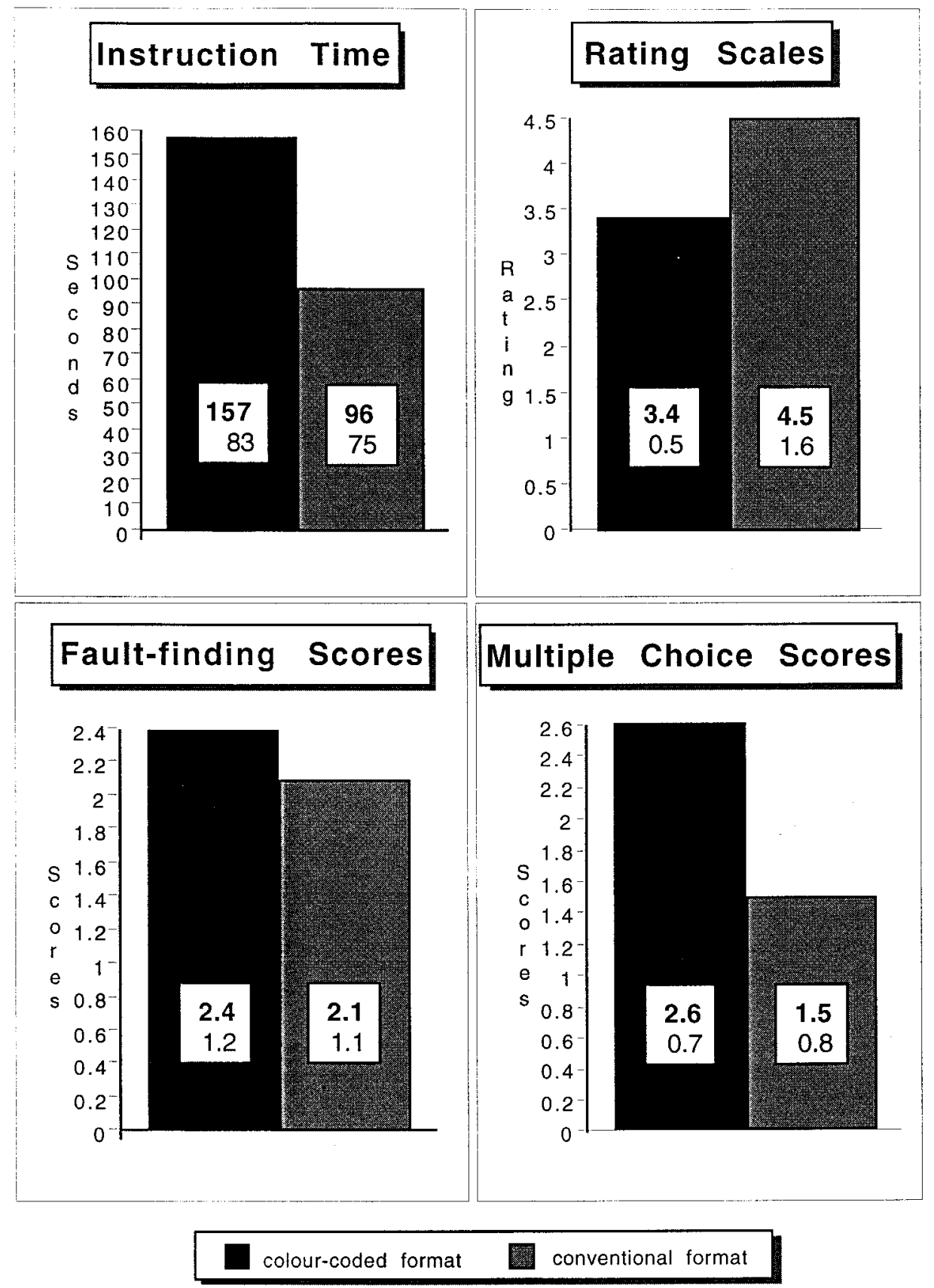

Figure 6. Charts of means for the data of Experiment 2

the circuit (i.e. Which element might be opened when the light is on? How can the light be turned off? Which element is normally closed? What is the switch needed for?). The fifth question consisted of a troubleshooting task where participants were presented with a textual description of a hypothetical problem in the circuit and 
were required to identify a possible cause of the problem (Which element might be out of order if the light goes off when the start button is released?). Four options were suggested (circuit breaker, start-button, stop-button, and switch) with the last option being correct. These questions provided a total score out of five for each participant. Up to 45 seconds were allowed for each question with the remaining time indicated on the screen.

\section{Results and discussion}

The variables under analysis were instruction time (time to process the instructions), subjective rating of mental load, and test performance scores on fault-finding and multiple-choice tasks. Means and standard deviations are displayed in the charts of Figure 6.

In order to take into account differences in learners' training times (the colourcoding group took more time to learn although the difference was non-significant, $F(1,13)=2.42, M S e=6246.63)$, analysis of covariance was applied to the data. The instruction times were used as a covariate in the analysis of covariance (ANCOVA). The results revealed significant differences between instructional groups on the multiple-choice items, $F(1,13)=7.17, M S e=0.61$. There was also a marginal difference between groups in subjective rating scale data, $F(1,13)=3.09, M S e=1.53$. No significant differences were found in fault-finding scores, $F(1,13)=0.78$, $M S e=1.29$. Absence of a significant difference in the fault-finding scores might be due to the task not necessarily requiring real understanding of the functioning of the circuit. Remembering the locations of the few elements of the circuit diagram might be sufficient to correctly answer the fault-finding problem.

Instructional effectiveness was calculated using Paas and Van Merrienboer's (1993) technique. Figure 7 demonstrates the results of the application of this procedure. The results for the colour-coding group are located in the high-effectiveness area (relatively lower cognitive load with higher performance), whereas the results for the conventional group are located in the area of low effectiveness (more cognitive load with lower performance). Analysis of covariance yielded a significant difference in effectiveness between the groups on the multiple-choice task, $F(1,13)=7.01, M S e=$ 1.12 , indicating that in terms of instructional effectiveness, the colour-coding format was by far more effective than the conventional format, as was predicted by cognitive load theory.

The colour-coded-diagram-and-text group demonstrated a lower subjective rating of cognitive load and higher test performance on multiple-choice items than the conventional group. The colour-coding technique can be used to eliminate the problems associated with split-attention. It should be considered as an alternative to physical integration and dual-mode presentation of split-source information.

The colour-coding technique might be especially appropriate in computer-based instructional presentations because they allow manageable use of colours. Using too many colours simultaneously may itself impose a significant load on working memory and eliminate any positive effect of colour-coding. Generally, graphic designers should not use more than about five distinct colours simultaneously (Marcus, 1991). Computer-based presentations allow designers to limit the concurrent use of many colours by using the techniques of Experiment 1 . 


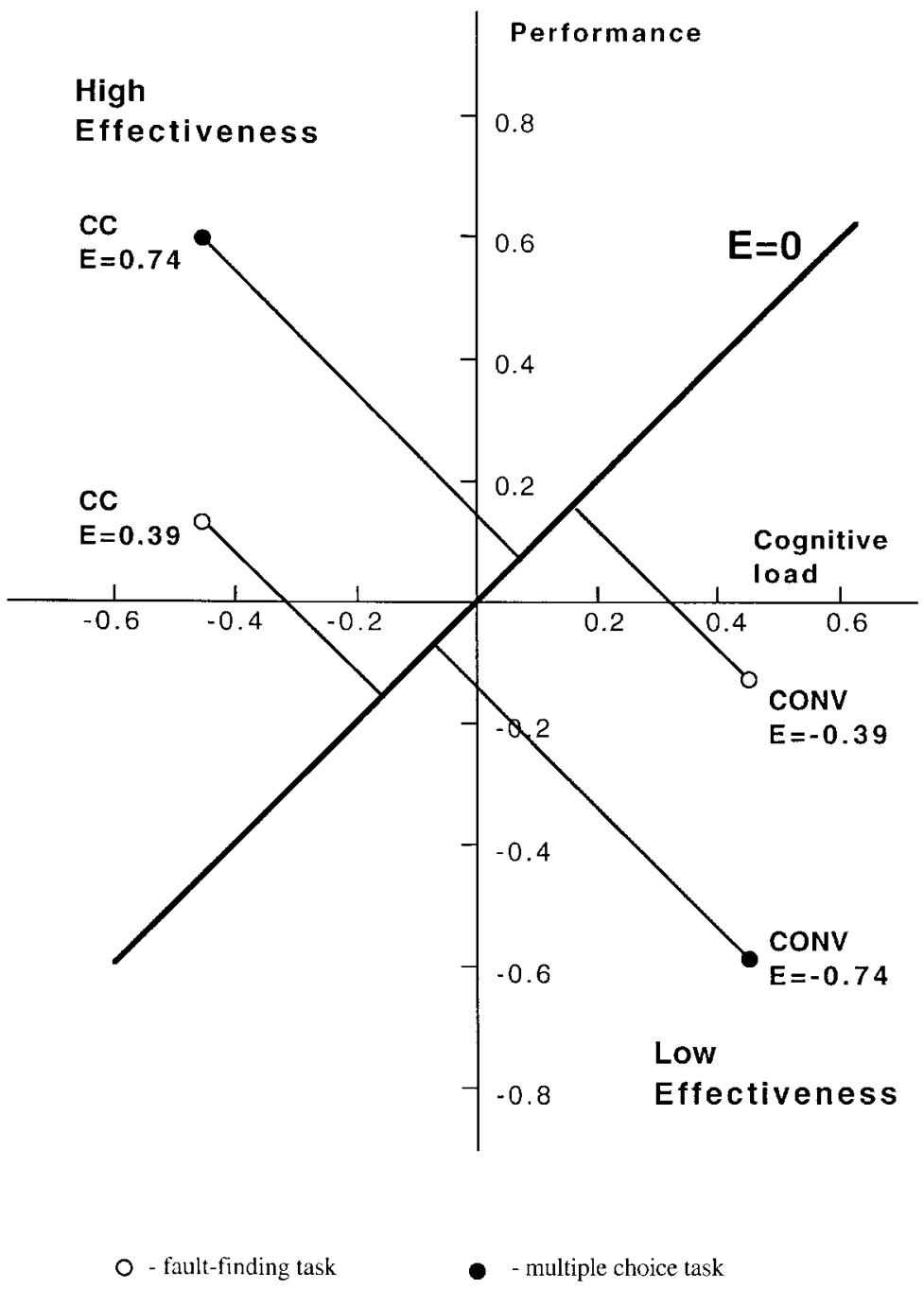

Figure 7. Representation of relative condition effectiveness $(E)$ for colour-coded-diagramand-text (CC) and conventional (CONV) groups for Experiment 2

\section{GENERAL DISCUSSION}

Human cognitive capacity is limited: we can process only a very limited amount of information at any one time. Cognitive load theory assumes that information presented to learners should be designed in a way to reduce any avoidable load on working memory. The experiments of this article were designed to test some ways of avoiding cognitive overload due to learners having to split their attention between text and diagrams.

Split-attention occurs when several sources of information are difficult or impossible to understand in isolation and must be mentally integrated to achieve 
understanding. If the sources of information are separated in space or time, learners must search for appropriate relations in order to mentally integrate the information. Search and matching related disparate information requires working memory resources that consequently are unavailable for schema acquisition, inhibiting learning. It was demonstrated in a number of previous studies that if the multiple sources of information are physically integrated, understanding can occur without an unnecessary search for relations between those sources of information. Cognitive load is reduced and schema acquisition is facilitated. The modality effect occurs under splitattention conditions, when separate sources of non-redundant information otherwise requiring mental integration are presented in alternate, auditory or visual, forms. Increasing effective working memory by using more than one sensory modality produces a positive effect on learning, similar to the effect of physically integrating separate sources of information.

Experiment 1 demonstrated that the modality effect may be used as a means of negating the problems associated with split-attention. A dual-mode instructional presentations resulted in superior learning compared with instructional materials presented in a split-source, visual-only format due to working memory capacity being enhanced under dual-modality conditions. Three computer-based multimedia instructional formats on theoretical aspects of soldering were compared (Visual text, Audio text, and Visual plus Audio text) using participants without any substantial knowledge of soldering. The Audio text group demonstrated a lower number of reattempts at interactive exercises, a lower subjective rating of cognitive load and higher test performance scores than each of the other two groups.

It is important to note that we are not proposing that audio text presentations always will be superior to equivalent visual-based presentations. We now will discuss some possible limitations. Audio text is unlikely to be effective if it is presented in such a way that it exceeds the processing capacity of limited working memory. For example, if the auditory component of instruction is too long or complex then it may overload working memory. Under these conditions, a visual text format may be more effective. Visual information frequently has the advantage over auditory information in being permanent and therefore can be referred to repeatedly while auditory information is fleeting and difficult to retrieve once heard. (Although audio repeat options are now available with most computer based multimedia presentations.)

The results of Experiment 1 confirmed the advantages of dual-mode presentations to overcome split-attention problems (Audio text group outperformed Visual text group), but they also demonstrated a disadvantage of dual-mode duplication of information (Audio text group outperformed Visual plus Audio text group). Inclusion of visually presented text simultaneously with the same text in an auditory form imposed an additional cognitive load. Elimination of redundant visual textual explanations proved to be beneficial. As noted in the introduction, it is common practice for educators and those involved in instructional design to use simultaneous visual and auditory text presentations when referring to diagrammatic information. The finding that such repetitive instructional formats may have serious negative consequences for learning suggest that a reconsideration of the structure of instructional materials such as multimedia presentations may be warranted. The elimination of simultaneously presented audio and visual text may assist in the 
learning process. This result can be related to the work of Schooler and EngstlerSchooler (1990) which demonstrated that verbalization of visual stimuli impaired subsequent recognition performance. The requirement to verbalize was redundant, producing cognitive overload.

The redundancy effect generally occurs under conditions where different sources of information are intelligible in isolation and where each source provides similar information but in a different form. Under these conditions, an unnecessary working memory load is caused not by learners having to split their attention between multiple sources of information but by the very existence of multiple sources of information. Attending to unnecessary information requires cognitive resources that consequently are unavailable for schema acquisition. Reducing cognitive load can be accomplished by eliminating redundant information thus freeing resources for learning.

Experiment 2 was performed to test colour-coding of text and diagrams as an alternative technique for circumventing the cognitive load consequences of splitattention. Colouring elements of a diagram in the same unique colours as corresponding textual elements was hypothesized to reduce an unnecessary working memory load by reducing search processes. Two computer-based instructional formats in elementary electrical engineering (conventional separate-diagram-and-text and colour-coded-diagram-and-text) were compared. The conventional format included an electrical circuit with a textual explanation written beneath the circuit. The colourcoded format consisted of exactly the same diagram and text, except that by clicking on any paragraph in the text all the electrical elements mentioned in that paragraph and depictions of those elements in the diagram were converted to the same unique colours.

The colour-coded format group demonstrated a marginally lower subjective rating of learning difficulty, providing an indicator of reduced working memory load. At the same time, this group demonstrated significantly higher test performance on multiplechoice items than the conventional format group. The subjective rating scales produced evidence that the effect was caused by cognitive load factors. Students found the colour-coded-diagram-and-text materials easier to process but performed at a higher level on the subsequent test, resulting in substantially higher instructional effectiveness ratings using Paas and Van Merrienboer's (1993) metric.

Both dual-mode presentation and colour-coding techniques were devised as alternatives to direct physical integration as a means of integrating information from text and diagrams. Both techniques proved effective and have clear and immediate implications for instructional design. Many multimedia instructional presentations are still based on common sense rather than theory or extensive empirical research. Visual formats tend to be determined purely by aesthetic considerations while the use of sound and its interaction with vision seems not to be based on any discernible principles. Using cognitive load reduction as an overarching principle, the current experiments suggest that when dealing with split-source diagrams and text (1) textual materials should be presented in auditory rather than written form; (2) textual materials should not be presented in both auditory and written form; (3) if textual materials must be presented in written form, search for diagrammatic referents should be reduced by using appropriate markers or guides such as colour-coding. These principles, based on cognitive load theory, can provide some guidance in the design of multi-media instruction. 


\section{ACKNOWLEDGEMENTS}

The work reported in this paper is supported by grants from the Australian Research Council (ARC) to Paul Chandler and John Sweller. We wish to thank our collaborating partners, Email Ltd and Webster Publishing for their ongoing support, and, in particular, Bryan Jones and Richard Winter from Email and Tony Webster from Webster Publishing.

\section{REFERENCES}

Baddeley, A. (1992). Working memory. Science, 255, 556-559.

Bobis, J., Sweller, J. and Cooper, M. (1993). Cognitive load effects in a primary school geometry task. Learning and Instruction, 3, 1-21.

Chandler, P. and Sweller, J. (1991). Cognitive load theory and the format of instruction. Cognition and Instruction, 8, 293-332.

Chandler, P. and Sweller, J. (1996). Cognitive load while learning to use a computer program. Applied Cognitive Psychology, 10, 1-20.

Chi, M. T. H., Glaser, R. and Rees, E. (1982). Expertise in problem solving. In R. Sternberg (Ed.) Advances in the Psychology of Human Intelligence (pp. 7-75). Hillsdale, NJ: Erlbaum.

Jeung, H., Chandler, P. and Sweller, J. (1997). The role of visual indicators in dual sensory mode instruction. Educational Psychology, 17, 329-343.

Kalyuga, S., Chandler, P. and Sweller, J. (1998). Levels of expertise and instructional design. Human Factors, 40, 1-17.

Larkin, J. H., McDermott, J., Simon, D. P. and Simon, H. A. (1980). Models of competence in solving physics problems. Cognitive Science, 4, 317-348.

Marcus, A. (1991). Graphic design for electronic documents and user interfaces. New York: ACM Press.

Mayer, R. (1989). Systematic thinking fostered by illustrations in scientific text. Journal of Educational Psychology, 81, 240-246.

Mayer, R. E. (1997). Multimedia learning: Are we asking the right questions? Educational Psychologist, 32, 1-19.

Mayer, R. and Anderson, R. (1991). Animations need narrations: An experimental test of a dual-coding hypothesis. Journal of Educational Psychology, 83, 484-490.

Mayer, R. and Anderson, R. (1992). The instructive animation: Helping students build connections between words and pictures in multimedia learning. Journal of Educational Psychology, 84, 444-452.

Mayer, R. and Gallini, J. (1990). When is an illustration is worth ten thousand words? Journal of Educational Psychology, 82, 715-726.

Mayer, R. E. and Sims, V. K. (1994). For whom is a picture worth a thousand words? Extensions of a dual-coding theory of multimedia learning. Journal of Educational Psychology, 86, 389-401.

Miller, G. A. (1956). The magical number seven, plus or minus two: Some limits on our capacity for processing information. Psychological Review, 63, 81-97.

Mousavi, S. Y., Low, R. and Sweller, J. (1995). Reducing cognitive load by mixing auditory and visual presentation modes. Journal of Educational Psychology, 87, 319-334.

Paas, F. and Van Merrienboer, J. (1993). The efficiency of instructional conditions: An approach to combine mental-effort and performance measures. Human Factors, 35, 737-743.

Paas, F. and Van Merrienboer, J. (1994). Variability of worked examples and transfer of geometrical problem-solving skills: A cognitive-load approach. Journal of Educational Psychology, 86, 122-133.

Paivio, A. (1990). Mental representations: A dual-coding approach. New York: Oxford University Press.

Penney, C. G. (1989). Modality effects and the structure of short term verbal memory. Memory and Cognition, 17, 398-422. 
Schneider, W. and Detweiler, M. (1987). A connectionist/control architecture for working memory. In G. H. Bower (Ed.), The psychology of learning and motivation (Vol.21, pp. 53-119).

Schooler, J. and Engstler-Schooler, T. (1990). Verbal overshadowing of visual memories: Some things are better left unsaid. Cognitive Psychology, 22, 36-71.

Sweller, J. (1994). Cognitive load theory, learning difficulty and instructional design. Learning and Instruction, 4, 295-312.

Sweller, J. and Chandler, P. (1994). Why some material is difficult to learn. Cognition and Instruction, 12, 185-233.

Sweller, J., Chandler, P., Tierney, P. and Cooper, M. (1990). Cognitive load factor in the structuring of technical material. Journal of Experimental Psychology: General, 119, $176-192$.

Sweller, J. van Merrienboer, J. and Paas, F. (1998). Cognitive architecture and instructional design. Educational Psychology Review, 10, 251-296.

Tarmizi, R. and Sweller, J. (1988). Guidance during mathematical problem solving. Journal of Educational Psychology, 80, 424-436.

Tindall-Ford, S., Chandler, P. and Sweller, J. (1997). When two sensory modes are better than one. Journal of Experimental Psychology: Applied, 3, 257-287. 